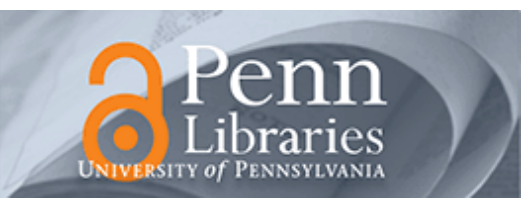

University of Pennsylvania ScholarlyCommons

November 2001

\title{
Filling Single Wall Carbon Nanotubes with Metal Chloride and Metal Nanowires and Imaging with Scanning Transmission Electron Microscopy
}

Satishkumar B. Chikkannanavar

University of Pennsylvania, satishkb@seas.upenn.edu

Andreas Taubert

University of Pennsylvania, taubert@seas.upenn.edu

David E. Luzzi

University of Pennsylvania, luzzi@Irsm.upenn.edu

Follow this and additional works at: https://repository.upenn.edu/mse_papers

\section{Recommended Citation}

Chikkannanavar, S. B., Taubert, A., \& Luzzi, D. E. (2001). Filling Single Wall Carbon Nanotubes with Metal Chloride and Metal Nanowires and Imaging with Scanning Transmission Electron Microscopy. Retrieved from https://repository.upenn.edu/mse_papers/19

Copyright Materials Research Society. Reprinted from MRS Proceedings Volume 706.

2001 Fall Meeting Symposium Z

Making Functional Materials with Nanotubes

Publisher URL: http://www.mrs.org/members/proceedings/fall2001/z/Z6_23.pdf

This paper is posted at ScholarlyCommons. https://repository.upenn.edu/mse_papers/19

For more information, please contact repository@pobox.upenn.edu. 


\title{
Filling Single Wall Carbon Nanotubes with Metal Chloride and Metal Nanowires and Imaging with Scanning Transmission Electron Microscopy
}

\author{
Abstract \\ Nanowires of magnetic metals $(\mathrm{Ho}, \mathrm{Gd})$ have been synthesized inside the hollow interior of single wall \\ carbon nanotubes by the sealed-tube reaction. Amongst the $\mathrm{d}$ - and $\mathrm{f}$-series metal chlorides investigated \\ in this study, $\mathrm{HoCl}_{3}$ and $\mathrm{GdCl}_{3}$ fill the SWNTs to a significantly higher extent than $\mathrm{FeCl}_{2}$ and $\mathrm{CoCl}_{2}$. $\mathrm{HoCl}_{3}$ \\ and $\mathrm{GdCl}_{3}$ nanowires have been transformed into the respective metal nanowires via the reduction of the \\ chloride nanowires. The nanowires have been imaged using high-resolution transmission electron \\ microscopy and scanning transmission electron microscopy (STEM). X-ray energy dispersive \\ spectroscopy carried out in conjunction with STEM confirmed the presence of metal chloride and metal \\ nanowires.

\section{Comments} \\ Copyright Materials Research Society. Reprinted from MRS Proceedings Volume 706. \\ 2001 Fall Meeting Symposium Z \\ Making Functional Materials with Nanotubes \\ Publisher URL: http://www.mrs.org/members/proceedings/fall2001/z/Z6_23.pdf
}




\title{
Filling Single Wall Carbon Nanotubes with Metal Chloride and Metal Nanowires and Imaging with Scanning Transmission Electron Microscopy
}

\author{
Satishkumar B. Chikkannanavar, Andreas Taubert and David E. Luzzi \\ Laboratory for Research on Structure of Matter, Department of Materials Science and \\ Engineering, University of Pennsylvania, Philadelphia, PA 19104-6272, USA
}

\begin{abstract}
Nanowires of magnetic metals (Ho, Gd) have been synthesized inside the hollow interior of single wall carbon nanotubes by the sealed-tube reaction. Amongst the d- and f-series metal chlorides investigated in this study, $\mathrm{HoCl}_{3}$ and $\mathrm{GdCl}_{3}$ fill the SWNTs to a significantly higher extent than $\mathrm{FeCl}_{2}$ and $\mathrm{CoCl}_{2}$. $\mathrm{HoCl}_{3}$ and $\mathrm{GdCl}_{3}$ nanowires have been transformed into the respective metal nanowires via the reduction of the chloride nanowires. The nanowires have been imaged using high-resolution transmission electron microscopy and scanning transmission electron microscopy (STEM). X-ray energy dispersive spectroscopy carried out in conjunction with STEM confirmed the presence of metal chloride and metal nanowires.
\end{abstract}

\section{INTRODUCTION}

Carbon nanotubes have been the subject of intense research because of their unique structure and electrical and mechanical properties [1,2]. Single wall carbon nanotubes (SWNTs) have been particularly interesting, since they have a uniform diameter and constitute ideal one-dimensional nanostructures. The hollow interior of SWNTs offers a one-dimensionally confined space that can be filled with various materials or be used to carry out chemical reactions. SWNTs have been filled with alkali halides $[3,4]$, among others. Various metal halides have been reduced within the confined space of the SWNTs, thus yielding metal nanowires [5]. In addition, molecular species like fullerenes and derivatives have also been filled inside SWNTs [6,7]. These filling processes lead to new composite materials with improved or different properties and functions. This is in part due to the one-dimensional nature of the species inside the carbon nanotubes. Magnetic nanowires based on SWNTs filled with ferromagnetic and other magnetic metals are a new and unexplored aspect of the nanotube filling that might prove to be potential for various applications.

In the present study, we have explored procedures to fill SWNTs with various dand f-metal chlorides as precursors for magnetic metal nanowires. This was done by a melt phase reaction or by a solution phase reaction of the chlorides with SWNTs. Later metal chloride nanowires are transformed into metal nanowires by annealing in hydrogen. Both the metal chloride and metal filled SWNTs were characterized using transmission electron microscopy (TEM), high resolution transmission electron microscopy (HRTEM), and scanning transmission electron microscopy (STEM) coupled with X-ray energy dispersive spectroscopy (XEDS). 
STEM relies on atomic number $(Z)$ contrast $[8,9]$ and the contrast directly scales as $Z^{2}$. As a result, STEM allows for the detection of high $Z$ elements such as $I_{2}$ in the low $Z$ environment of the carbon nanotubes [10]. Here, we have used bright field (BF) and high angle annular dark field (HAADF) STEM to confirm the formation of high Z Hoand Gd-nanowires within the carbon nanotubes. XEDS confirmed the reduction of metal chloride to the metal nanowires.

\section{EXPERIMENTAL}

The SWNTs used in the present filling experiments were carbon arc produced, using Y/Ni as the catalyst. As-received SWNTs were purified by a combination of air oxidation and acid treatment. Air oxidation was carried out in static air at $360^{\circ} \mathrm{C}$ for $3 \mathrm{~h}$ followed by refluxing the SWNTs in $5.3 \mathrm{M} \mathrm{HNO}_{3}$ for $40 \mathrm{~min}$, and subsequent neutralization with $\mathrm{NaOH}$ at $\mathrm{pH}$ 10. The SWNTs were filtered on a Nylon filter paper with repeated washing with deionized water. After resuspension in toluene with sonication, they were filtered on a Teflon paper and dried at $115^{\circ} \mathrm{C}$ overnight.

For the melt-phase filling, $\sim 2-3 \mathrm{mg}$ of so purified SWNTs were mixed with excess $(\sim 20 \mathrm{mg})$ of the respective metal chloride $\left(\mathrm{FeCl}_{2}, \mathrm{CoCl}_{2}, \mathrm{GdCl}_{3}\right.$, and $\mathrm{HoCl}_{3}$; from Aldrich), with gentle grinding. The mixture was inserted into a quartz ampoule, evacuated to $10^{-2}$ torr and sealed. The vacuum tight quartz ampoules were heat-treated at high temperatures (Table 1) for both 24 and $48 \mathrm{~h}$. The temperatures for heat treatment were chosen such that they were $\sim 100^{\circ} \mathrm{C}$ higher than the respective melting temperatures of the metal chlorides. For the solution-phase filling, $\sim 2 \mathrm{mg}$ of the SWNTs and $\sim 20 \mathrm{mg}$ of the metal chloride(s) were refluxed in $\sim 5 \mathrm{ml}$ of $12 \mathrm{M} \mathrm{HCl}$ for $2-3$ hours. The sotreated SWNTs were washed with deionized water in order to remove the exterior chloride and dried in an oven.

Samples of filled SWNTs were dispersed in ethanol, sonicated, and drop cast onto holey carbon copper grids. Transmission electron microscopy experiments were done in a JEOL 2010F field emission transmission electron microscope. Bright field and highresolution imaging were carried out at $100 \mathrm{kV}$. Images were acquired on photographic film and lattice planes were measured from the negatives, taking the graphene (002) planes as reference. Scanning transmission electron microscopy (STEM) was carried out in the same microscope at $200 \mathrm{kV}$ with a 0.2 or $0.5 \mathrm{~nm}$ probe, using a Gatan bright field (BF) and a JEOL high angle annular dark field (HAADF) detector. We used a $50 \mu \mathrm{m}$ condensor aperture and a dark field collection angle of $\sim 80 \mathrm{mrad}$. Image size was $512 \mathrm{x}$ 408 pixels, magnifications ranged from $\sim 150,000 \mathrm{X}$ to $1,500,000 \mathrm{X}$, with image acquisition time of $21 \mathrm{~s}$. X-ray energy dispersive spectroscopy (XEDS) was done with a Princeton Gamma Tech (PGT) XEDS spectrometer with a $\mathrm{Si}(\mathrm{Li})$ detector and data analysis was performed with the IMIX software from PGT.

\section{RESULTS AND DISCUSSION}

The solution phase filling resulted in sparsely filled SWNTs with the metal chlorides. The fact that solution-phase filled nanotubes are only sparsely filled, implies that solution-phase filling is not suitable for achieving high filling fractions. Since for the investigation of the properties of SWNT/metal nanocomposites a high filling fraction is desired, we did not further pursue the solution-phase filling process. Previous melt phase 
filling experiments resulted in continuously filled SWNTs with high filling fractions $[3,5]$. We therefore decided to investigate the filling from the melt. The melting temperatures and the heat treatment temperatures for the various metal chlorides (for dand f-series metals) used in our study are displayed in Table 1.

\begin{tabular}{ccc}
\hline Metal Chloride & $\begin{array}{c}\text { Melting Temperature } \\
\left({ }^{\circ} \mathrm{C}\right)\end{array}$ & $\begin{array}{c}\text { Reaction Temperature } \\
\left({ }^{\circ} \mathrm{C}\right)\end{array}$ \\
\hline $\mathrm{HoCl}_{3}$ & 718 & 820 \\
$\mathrm{FeCl}_{2}$ & 674 & 770 \\
$\mathrm{GdCl}_{3}$ & 609 & 700 \\
$\mathrm{CoCl}_{2}$ & 724 & 820 \\
$\mathrm{FeCl}_{2}+\mathrm{CoCl}_{2}$ & --- & 800 \\
$\mathrm{HoCl}_{3}+\mathrm{FeCl}_{2}$ & --- & 800 \\
$\mathrm{HoCl}_{3}+\mathrm{CoCl}_{2}$ & --- & 800 \\
\hline
\end{tabular}

Table 1. Melting temperature and reaction temperature for various metal chlorides for melt phase filling experiments

Amongst the metal chlorides studied, the highest filling fraction from the melt phase was observed with $\mathrm{HoCl}_{3}$ and $\mathrm{GdCl}_{3}(\sim 60 \%$ of SWNT ropes being filled, based on detailed TEM observations). A HRTEM image of a rope of SWNTs filled with $\mathrm{HoCl}_{3}$ is shown in Fig. 1. The chloride nanowires have a diameter of $\sim 1 \mathrm{~nm}$ and run along the long axis of SWNTs (arrows). The nanowires extend over several $100 \mathrm{~nm}$ without any breaks or interruptions. The lattice planes of the $\mathrm{HoCl}_{3}$ nanowires in the HRTEM image (Figure 1) exhibit a preferred orientation, with planes being perpendicular to the long axis of nanotube, and have a spacing of $\sim 3.45 \AA$. We believe that these planes are associated with the $\mathrm{HoCl}_{3}$ lattice, since no crystal structure has been reported for $\mathrm{HoCl}_{3}$ yet. Figure 1 also shows that in addition to nanowires located within the nanotubes, the chloride is also located at the exterior to the nanotubes and ropes.

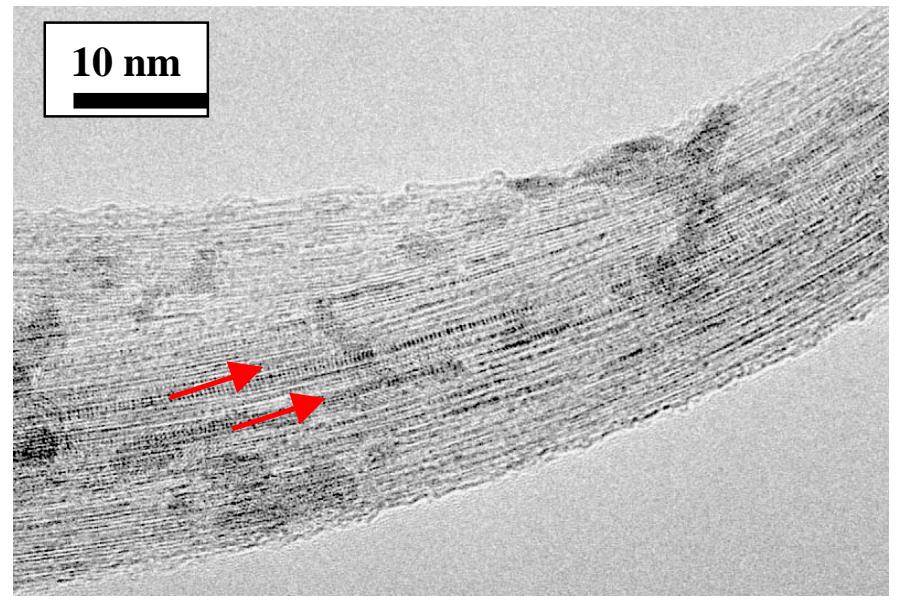

Figure 1. HRTEM image of a large rope of SWNTs filled with $\mathrm{HoCl}_{3}$ nanowires. The lattice plane spacing is $\sim 3.45 \AA$. In addition to nanowires the chloride is seen on the surface of SWNT rope.

The different filling characteristics of the $\mathrm{d}$ - and f-metal chlorides may be due to the difference in the wetting property of the various chlorides. Only materials with a low surface tension $(<200 \mathrm{mN} / \mathrm{m})$ are expected to fill (or wet) the interior of carbon nanotubes [11]. Since $\mathrm{HoCl}_{3}$ and $\mathrm{GdCl}_{3}$ fill the SWNTs, we may conclude that the 
surface tension of these f-metal chlorides is lower than one of the d-element chlorides investigated here.

The SWNTs filled with holmium and gadolinium chloride were annealed in hydrogen atmosphere at $550^{\circ} \mathrm{C}$ for $8 \mathrm{~h}$. This transformed the chloride nanowires into the respective metal nanowires. The Ho and Gd nanowires obtained from this process are intact and continuous along the length of the carbon nanotubes (Figure 2). The metal nanowires show a preferred orientation with respect to the axis of the carbon nanotubes. The measured lattice spacing from the HRTEM images is $~ 3.1 \AA$, which corresponds to the (100) planes of hexagonal close packed (hcp) holmium (jcpds file: 02-0910). Selected area diffraction (SAD) patterns recorded on the Ho nanowire-filled SWNT ropes showed diffuse ring patterns (inset in Figure 2). Taking the graphene (100) reflection with $\mathrm{d} \sim 2.1$ $\AA$ (shown as $(100) \mathrm{G}$ ) as the internal standard, the ring patterns from the SWNT/metal nanowire composite was determined to originate from lattice planes with $\mathrm{d} \sim 3.1 \AA$ which is consistent with Ho (100) lattice plane spacings.

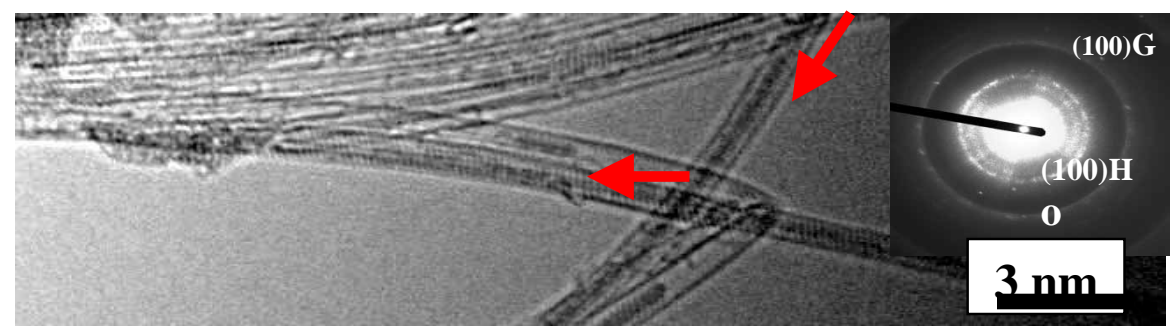

Figure 2. HRTEM image of holmium nanowires filled inside the SWNTs obtained after hydrogen anneal of nanotubes filled with chloride at $550^{\circ} \mathrm{C}$ for $8 \mathrm{~h}$. The lattice plane spacing is $\sim 3.1 \AA$, representing the (100) planes of hcp holmium.
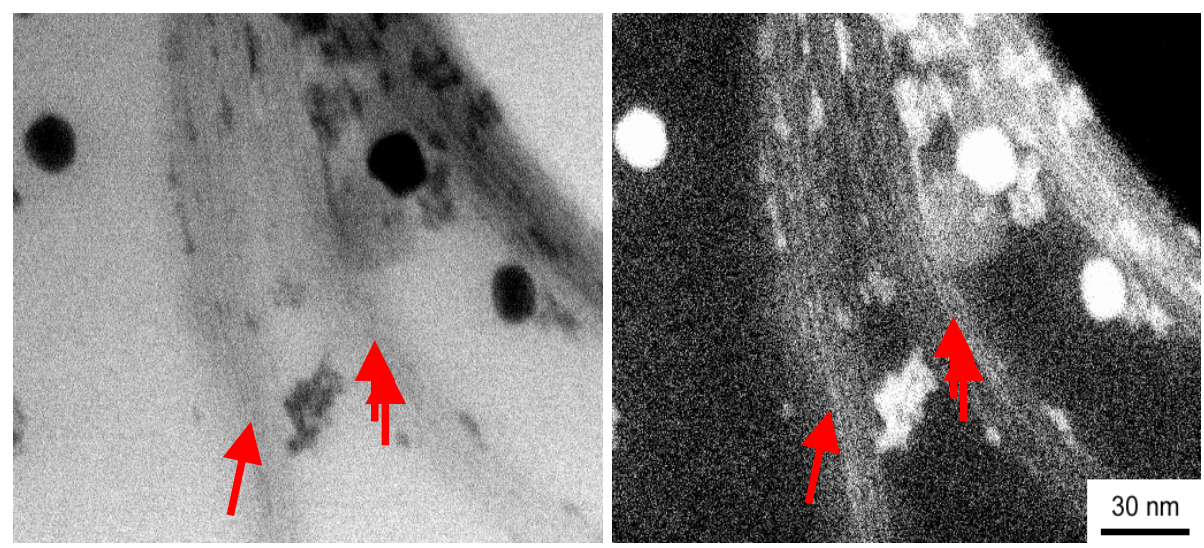

Figure 3. STEM bright field (left) and annular dark field (right) image pair of $\overline{\mathrm{HoCl}}_{3}$ filled SWNT ropes showing the dark contrast due to $\mathrm{Z}$-specific scattering from holmium and chlorine atoms.

STEM confirmed the presence of continuous nanowires with a high average atomic number $\mathrm{Z}$ within the nanowires. This was concluded from the fact that between the bright field $(\mathrm{BF})$ and the high angle annular dark field (HAADF) images, contrast inversion occurred for both the metal chloride (Figure 3, $\mathrm{HoCl}_{3}$ ) and the metal samples after hydrogen annealing (Figure 4, Ho). The dark lines in the BF image and the bright lines in the HAADF image correspond to the nanowires incorporated in the SWNTs. The extraneous larger features are considered to be due to chloride impurities from the sample preparation along with $\mathrm{Ni} / \mathrm{Y}$ catalyst particles. 

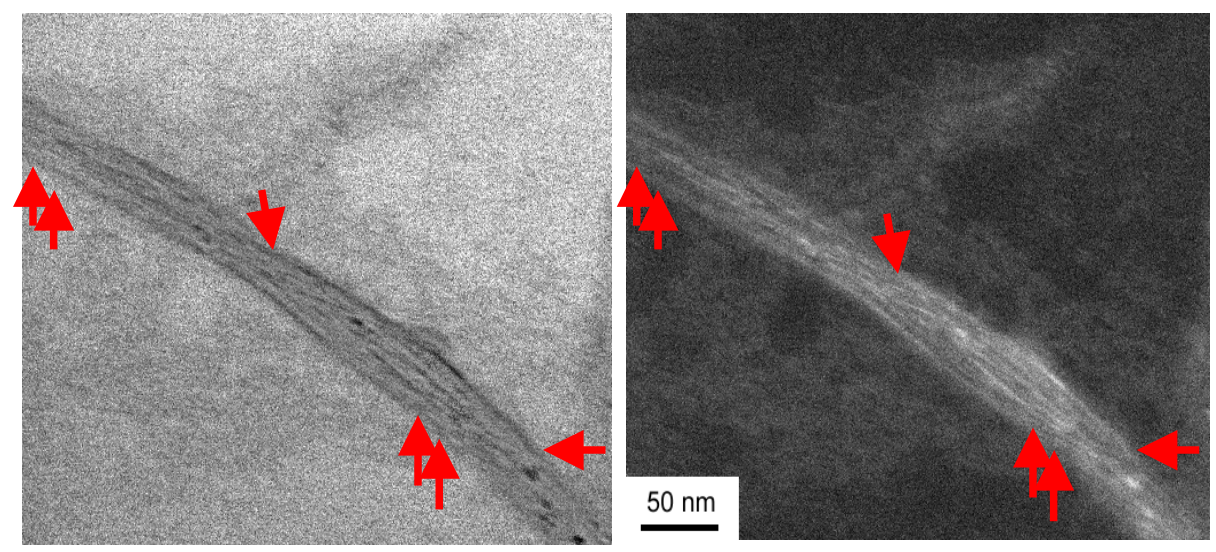

Figure 4. STEM bright field (left) and dark field (right) image pair of the SWNT rope filled with nanowires of Ho. The nanowires are more evident on the edges compared to the middle portion of the rope.

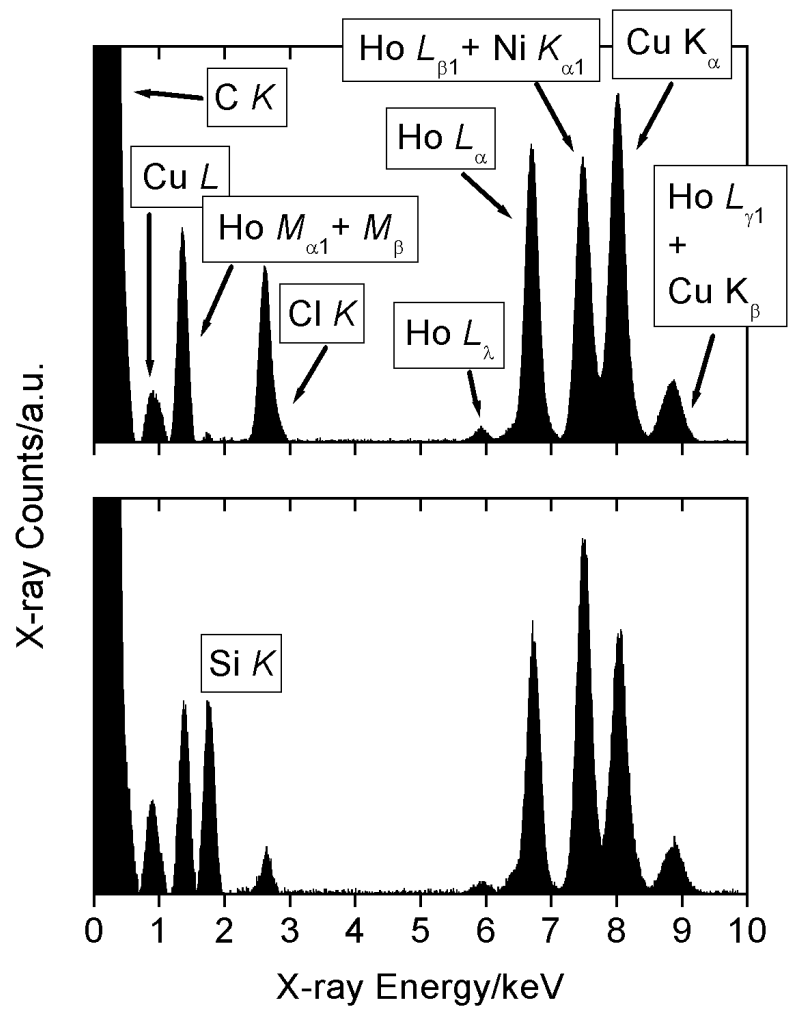

Figure 5. XEDS spectra recorded on SWNT ropes filled with $\mathrm{HoCl}_{3}$ (top) and $\mathrm{Ho}$ nanowires (bottom) showing the presence of $\mathrm{Ho}$ and $\mathrm{Ho}, \mathrm{Cl}$ signals respectively, in addition to catalyst and copper peaks. The area of coverage was $\sim 1 \mathrm{~mm}^{2}$. The weak chlorine peak in case of Ho nanowires is due to the large exterior particles, which have not been reduced completely to the metal.

The presence of the holmium chloride and holmium was further confirmed by means of X-ray energy dispersive spectroscopy (Figure 5). The spectra were recorded from ropes of filled SWNTs but spectra taken from the Ho and Gd-filled SWNTs showed the Ho or Gd along with Ni, Y, C- $K$, and $\mathrm{Cu}-K$ and $L$ lines. For the chlorides, an intense $\mathrm{Cl}$ signal was observed in addition to other peaks. The $\mathrm{Y}$ and Ni peaks are due to the presence of catalyst particles as seen in the BF and HAADF STEM images as spherical features with nanometer dimensions. The $\mathrm{Cu}$ peaks are due to the copper grid, and $\mathrm{Si}-K$ 
peak is due to holey carbon support grid. The $\mathrm{C}$ signal is due to the support carbon film and the SWNTs. Gd, Ho, and $\mathrm{Cl}$ lines originate from the respective nanowires and exterior metal chloride on the nanotube surface. These data therefore confirm that the samples contain either $\mathrm{MCl}_{3}$ or $\mathrm{M}(\mathrm{M}=\mathrm{Ho}, \mathrm{Gd})$, respectively.

\section{CONCLUSIONS}

Nanowires of f-metal chlorides have been successfully filled inside the interiors of SWNTs through a melt phase reaction. The solution phase filling, on the other hand, has been found to poorly fill the SWNTs, compared to the melt phase method. The chloride nanowires have been transformed to metal nanowires by annealing in hydrogen atmosphere. TEM, HRTEM and SAD analysis confirmed the formation of chloride and metal nanowires. XEDS spot analysis in conjunction with BF/HAADF STEM imaging confirmed the formation of nanowires. As a result, our experiments demonstrate that SWNTs can be filled with magnetic materials thereby forming an entirely new class of materials. Our paper also demonstrates that the combination of TEM, HRTEM, and analytical STEM is capable of providing structural and chemical information about SWNT/nanowire composites not accessible with other techniques.

\section{ACKNOWLEDGEMENTS}

The authors thank Dr. Doug M. Yates (University of Pennsylvania) for technical assistance with the JEOL 2010F and Prof. P. Bernier (CNRS, Montpellier, France) for the SWNT sample. Financial support from the Office of Naval Research grant is gratefully acknowledged.

\section{REFERENCES}

[1] Fullerenes: Chemistry, Physics and Technology, Edrs. K. M. Kadish, and R. S. Ruoff, John Wiley \& Sons (2000)

[2] C. N. R. Rao et al., Chem. Phys. Chem. 2, 78 (2001)

[3] J. Sloan et al., Chem. Commun. 699 (1999)

[4] R. R. Meyer et al., Science 289, 1324 (2000)

[5] A. Govindaraj et al., Chem. Mater. 12 202, (2000)

[6] B. W. Smith, M. Monthioux and D. E. Luzzi, Nature 363, 603 (1998)

[7] R. Russo et al., presented at MRS Spring Meeting 675, w1.3.1-1.3.7 (2001)

[8] P. D. Nellist and S. J. Pennycook, Phys. Rev. Lett. 81, 4156 (1998)

[9] A. V. Kadanavich et al., J. Phys. Chem. 105, 361 (2001)

[10] X. Fan et al., Phys. Rev. Lett. 84, 4621, (2000)

[11] E. Dujardin et al., Adv. Mater. 101472 (1998) 